\title{
The Dose-Response Association Between Reported Moderate to Vigorous Intensity Physical Activity and Atherogenic Index of Plasma: NHANES, 1999-2006
}

\author{
Meghan Edwards and Paul Loprinzi
}

\begin{abstract}
Objective: Atherogenic index of plasma (AIP), calculated as $\mathrm{LOG}_{10}$ (triglycerides/high-density lipoprotein-cholesterol), may be utilized to predict risk for cardiovascular disease. Physical activity has been shown to have a favorable association with triglycerides and high-density lipoprotein-cholesterol. The dose-response association between moderate to vigorous physical activity (MVPA) and AIP has yet to be determined in a nationally representative sample of US adults, which was the purpose of this study. Methods: Data from the 1999-2006 National Health and Nutrition Examination Survey were used (N=6694 adults aged 20-85 y). Leisure-time physical activity over the previous 30 days was self-reported, whereas AIP was calculated from a venous blood sample. Multivariable linear and logistic regression tested the independent association between MVPA and AIP. Results: The authors observed a significant inverse, graded association between quantile of MVPA and the AIP $\left(P_{\text {trend }}<.001\right)$. Conclusion: Engaging in MVPA may attenuate the risk for cardiovascular disease via improvements in AIP. More longitudinal research is necessary to confirm these cross-sectional findings.
\end{abstract}

Keywords: aerobic exercise, epidemiology, lipoprotein, triglycerides

The atherogenic index of plasma (AIP), calculated as $\mathrm{LOG}_{10}$ (triglycerides/high-density lipoprotein-cholesterol [HDL-C]), ${ }^{1}$ is directly associated with cardiovascular disease (CVD) risk. ${ }^{2-4}$ Triglycerides and HDL-C are both measured during a traditional lipid profile assessment, making this a feasible measure to employ in the clinical setting. Notably, AIP has been shown to be a better predictor of CVD risk when compared with other atherogenic indicies ${ }^{3,5}$ or lipids alone. ${ }^{4}$ The potential utility of AIP as a marker for CVD underscores the importance of exploring various independent factors associated with AIP, in order to develop and implement the most effective risk reduction strategies.

Physical activity has been shown to favorably associate with HDL-C ${ }^{6}$ and triglycerides ${ }^{7}$ and is well understood to inversely associate with CVD risk. ${ }^{89}$ Recent experimental work has also demonstrated an inverse relationship between aerobic exercise and AIP. ${ }^{10}$ However, some intervention-based research investigating the exercise-AIP relationship has presented mixed results. For instance, an 8-week continuous aerobic exercise intervention significantly reduced AIP in young adult men, whereas an 8-week interval-based exercise intervention did not. ${ }^{11}$ Similarly, a 12 -week aerobic training program in adult men resulted in significantly decreased AIP, whereas a 12-week resistance-training program yielded no significant changes. ${ }^{12}$ Given the paucity of research evaluating the associations of modifiable behaviors (eg, physical activity) with AIP and the previously mentioned mixed results of experimental work on physical activity and AIP, the purpose of the present brief report was to examine the dose-response association of moderate to vigorous physical activity (MVPA) and AIP among a nationally representative sample of US adults. Such a study, to the authors' knowledge, has yet to be conducted. Examining this doseresponse association within a nationally representative sample

The authors are with the Department of Health, Exercise Science and Recreation Management, The University of Mississippi, University, MS. Loprinzi (pdloprin@ olemiss.edu) is corresponding author. will increase the evidence supporting a biological link between the physical activity and the AIP and will maximize the generalizability of the authors' findings beyond those of the previously mentioned intervention studies.

\section{Methods}

\section{Study Design and Participants}

Data from the 1999-2006 National Health and Nutrition Examination Survey (NHANES) were analyzed for this report. Written informed consent was obtained from all participants. The NHANES is an ongoing survey conducted by the Center for Disease Control and Prevention designed to evaluate the health status of US adults through a complex, multistage, stratified clustered probability design. Further details of NHANES can be found elsewhere (http://www.cdc.gov/nchs/nhanes.htm). Participants included 6694 adults (aged 20-85 y) with complete data on the study variables.

\section{Physical Activity}

As described elsewhere, ${ }^{13}$ participants were asked open-ended questions about participation in leisure-time physical activity over the past 30 days. Data were coded into 48 activities, including 16 sports-related activities, 14 exercise-related activities, and 18 recreational-related activities. For each of the 48 activities, where participants reported MVPA for the respective activity, they were asked to report the number of times they engaged in that activity over the past 30 days and the average duration they engaged in that activity.

For each activity, metabolic equivalent of task $([\mathrm{MET}] \cdot \mathrm{min} /$ mo) was calculated by multiplying the number of days, by the mean duration, by the respective MET level $($ MET $\cdot \mathrm{min} / \mathrm{mo}=\mathrm{d} \times$ duration $\times$ MET level). The MET levels for each activity are provided 
elsewhere. ${ }^{14,15}$ Participants engaging in $\geq 2000$ MET $\cdot$ minute per month of leisure-time physical activity were defined as meeting physical activity guidelines. This is based on recommendations from the Office of Disease Prevention and Health Promotion, ${ }^{16}$ indicating that weekly physical activity of at least $500 \mathrm{MET} \cdot$ minute per month is consistent with government MVPA guidelines and sufficient for producing substantial health benefits. As described elsewhere, ${ }^{13}$ this self-reported physical activity measure has demonstrated evidence of convergent validity by associating with accelerometer-determined physical activity.

\section{Atherogenic Index of Plasma}

The AIP was determined from a venous blood sample and calculated as: $\mathrm{LOG}_{10}$ (triglycerides/HDL-C), with triglycerides $(\mathrm{mg} / \mathrm{dL} /$ 88.57) and HDL-C (mg/dL/38.67) expressed in mmol/L. Based on the manner of calculation, values for the AIP can range below and above 0. For example, if someone had a triglyceride level of $110 \mathrm{mg} / \mathrm{dL}$ and an HDL level of $90 \mathrm{mg} / \mathrm{dL}$, their AIP would be $-0.273 \mathrm{mmol} / \mathrm{L}$ (LOG[110 $\mathrm{mg} / \mathrm{dL} / 90 \mathrm{mg} / \mathrm{Dl}]=-0.273$ ). The AIP was expressed as a continuous variable, whereas AIP $>24 \mathrm{mmol} / \mathrm{L}$ was considered elevated. ${ }^{2}$ This threshold was based on the association of AIP with various clinical outcomes, such as hypertension, diabetes, and dyslipidemia. ${ }^{2}$

\section{Statistical Analysis and Covariates}

Both MVPA and the AIP were used as continuous and categorical variables in the multivariable linear and logistic regression analyses. All analyses adjusted for the NHANES complex, multistage probability design. In all models, the following covariates were included age, sex, race/ethnicity, physician-diagnosed hypertension, totaland low-density lipoprotein-cholesterol concentrations, body mass index, smoking status, and cholesterol-lowering medication. A multiplicative interaction between MVPA and each covariate was tested by creating a cross-product term and including it, along with the main effects and the covariates, in the regression modeling. In order to determine whether there was a dose-response association between MVPA and AIP, quantiles of MVPA were created $(\mathrm{Q} 1=0, \mathrm{Q} 2=444-491, \mathrm{Q} 3=2457-2596$, and Q4 = 12,711-14,835 $\mathrm{MET} \cdot \mathrm{min} / \mathrm{mo})$. Findings from the regression modeling were expressed as adjusted parameter estimates $(\beta)$ or odds ratios (ORs), along with their $95 \%$ confidence interval (CI). All statistical analyses were performed using Stata (v 12; College Station, TX), with an alpha level set at $\alpha<.05$.

\section{Results}

Weighted characteristics of the analyzed sample are shown in Table 1. Participants, on average, were 44 years of age and mostly white, with an equal number of men and women. About $47 \%$ of the participants met MVPA guidelines based on their reported physical activity. In the multivariable linear regression modeling, those participants who reported $\geq 2000 \mathrm{MET}$-minute per month of MVPA had a significantly lower AIP than those who did not $\left(\beta_{\text {adjusted }}=-0.04 \mathrm{mmol} / \mathrm{L} ; 95 \% \mathrm{CI},-0.06\right.$ to $\left.-0.02 \mathrm{mmol} / \mathrm{L}\right)$. Moreover, those meeting MVPA guidelines experienced a 33\% lower odds of having an elevated AIP than their less-active counterparts $(\mathrm{OR}=0.67 ; 95 \% \mathrm{CI}, 0.57$ to 0.79$)$. These findings were not influenced by sociodemographic characteristics, hypertension, total- or low-density lipoprotein-cholesterol concentrations, body mass index, smoking status, or cholesterol-lowering
Table 1 Weighted Characteristics of the Analyzed Sample: NHANES, 1999-2006 ( $=6694)$

\begin{tabular}{lcc}
\hline Variable & Point estimate & $\mathbf{9 5 \%} \mathbf{C l}$ \\
\hline AIP, mean mmol/L & -0.02 & -0.03 to -0.009 \\
$\quad$ \%Elevated $(>0.24)$ AIP & 20.2 & \\
Age, mean y & 44.1 & 43.3 to 44.8 \\
\%Female & 52.0 & \\
\%White & 70.8 & \\
\%Meeting MVPA guidelines & 46.6 & \\
Total cholesterol, mean mg/dL & 199.0 & 197.6 to 200.4 \\
LDL-C & 119.9 & 118.6 to 121.1 \\
Body mass index, mean $\mathrm{kg} / \mathrm{m}^{2}$ & 28.0 & 27.7 to 28.2 \\
\%Physician-diagnosed hypertension & 23.7 & \\
\%Smoker & 23.5 & \\
\%On cholesterol medication & 8.4 & \\
\hline
\end{tabular}

Abbreviations: AIP, atherogenic index of plasma; CI, confidence interval; LDL-C, low-density lipoprotein-cholesterol; MVPA, moderate to vigorous physical activity; NHANES, National Health and Nutrition Examination Survey.

medication. Moreover, the independent association between MVPA and AIP did not vary by level of any covariate, as none of the interaction terms achieved statistical significance.

As indicated by unadjusted data in Figure 1, the authors observed a significant inverse, graded association between quantile of MVPA and the AIP $\left(P_{\text {trend }}<.001\right)$. After adjustment for the covariables of interest, those participants in Q2 (444-491 MET·min/mo) had an AIP that was $0.038 \mathrm{mmol} / \mathrm{L}$ lower $(95 \% \mathrm{CI},-0.06$ to $-0.01 \mathrm{mmol} / \mathrm{L})$ than participants in the bottom quantile (the referent group). Participants in Q3 (2457-2596 MET·min/mo) lowered their AIP by $0.033 \mathrm{mmol} / \mathrm{L}$ (95\% CI, -0.05 to $-0.01 \mathrm{mmol} / \mathrm{L})$, whereas the most active participants in Q4 (12,711-14,835 MET.min/month) had an AIP about $0.063 \mathrm{mmol} / \mathrm{L}$ lower $(95 \% \mathrm{CI},-0.08$ to $-0.04 \mathrm{mmol} / \mathrm{L})$ compared with the referent group. In terms of cardiovascular risk, the authors observed about a 30\% lower odds of an elevated AIP in Q2 (OR = $0.71 ; 95 \% \mathrm{CI}, 0.52$ to 0.96 ), and Q3 (OR $=0.72 ; 95 \% \mathrm{CI}, 0.60$ to $0.86)$ compared with the referent group, whereas those participants reporting the highest level of MVPA lowered their odds by $42 \%$ $(\mathrm{OR}=0.58 ; 95 \% \mathrm{CI}, 0.49$ to 0.68$)$.

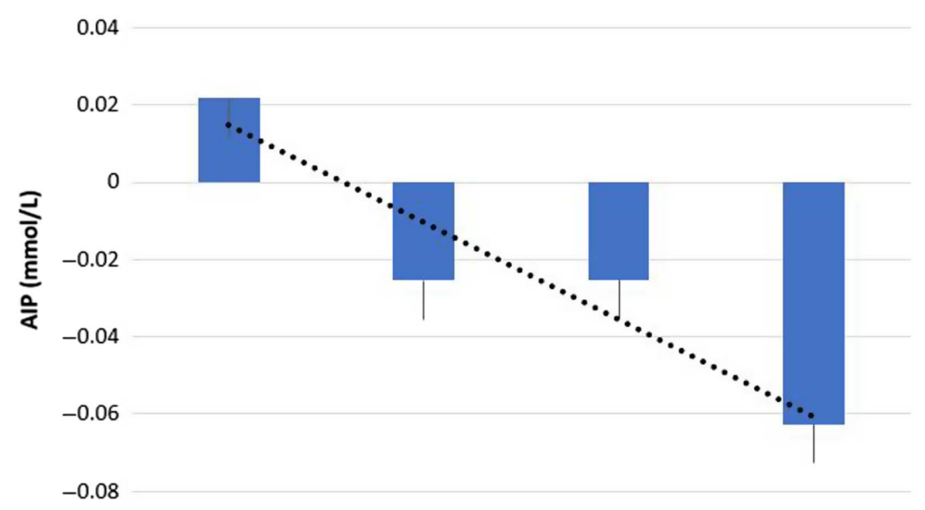

Figure 1 - Unadjusted association between quantile (Q) of moderate to vigorous physical activity (MVPA) (MET-min/mo) and the atherogenic index of plasma (AIP, mmol/L). Data represent the mean (SE) of AIP across quantiles of MVPA. Dotted line represents the trend line $(P<.001)$. MVPA Q1 $=0$, Q2 $=444$ to $491, \mathrm{Q} 3=2457$ to 2596 , and Q4 $=12,711$ to 14,835 MET.minute per month. 


\section{Discussion}

The AIP is a lipid profile ratio that may serve as a marker for chronic conditions related to atherosclerosis (eg, CVD). ${ }^{2-4}$ In accordance with a previous study, ${ }^{10}$ the authors observed an inverse association between MVPA and AIP. Specifically, participants who reported $\geq 2000 \mathrm{MET} \cdot \mathrm{minute}$ per month of MVPA had a significantly lower odds of having an elevated AIP ( $>24 \mathrm{mmol} / \mathrm{L})$, and these findings were independent of a number of important sociodemographic and clinical variables. This study extends previous findings by demonstrating a significant, inverse dose-response association between quantile of MVPA and both level of AIP and the risk of an elevated AIP.

The present findings, though strengthened by the use of a large and nationally representative sample, should be interpreted with consideration of the limitations of a cross-sectional study design. It is possible that individuals with a higher AIP level may choose to engage in less MVPA as a result of certain physical limitations. However, the implied conclusion that inadequate levels of MVPA may result in a higher AIP, is also plausible, particularly given the previous intervention work on this topic. ${ }^{11,12}$

Mechanistically, there is still much to learn regarding the exercise-lipid profile relationship. ${ }^{17}$ Notably, there is some evidence that MVPA promotes the preferential utilization of lipids over glycogen for fuel, ${ }^{18}$ resulting in lower circulating lipid levels. ${ }^{17}$ Exercise has also been postulated to alter the rates of key enzyme reactions (eg, cholesterol ester transfer protein) involved in cholesterol esterification, ${ }^{19}$ which correlates with AIP. ${ }^{20}$

\section{Conclusion}

The main findings of this brief report indicate that adults who engaged in recommended levels of MVPA had a reduced odds of elevated AIP, and that these benefits may occur in a dose-response manner. Along with additional mechanistic research, future longitudinal studies on the current topic are needed to confirm our findings. A continued investigation, via experimental interventions, of the differential effects of varying durations, intensities, and types of exercise on AIP would also be beneficial for providing the most effective CVD prevention and risk reduction strategies.

\section{References}

1. Dobiasova M, Frohlich J. The plasma parameter log (TG/HDL-C) as an atherogenic index: correlation with lipoprotein particle size and esterification rate in apoB-lipoprotein-depleted plasma (FER(HDL)). Clin Biochem. 2001;34(7):583-588. PubMed ID: 11738396 doi:10. 1016/S0009-9120(01)00263-6

2. Dobiasova M. AIP-atherogenic index of plasma as a significant predictor of cardiovascular risk: from research to practice. Vnitr Lek. 2006;52(1):64-71. PubMed ID: 16526201

3. Bhardwaj S, Bhattacharjee J, Bhatnagar M, Tyagi S. Atherogenic index of plasma, castelli risk index and atherogenic coefficient: new parameters in assessing cardiovascular risk. Int J Pharm Biol Sci. 2013;3(3):359-364.

4. Essiarab F, Taki H, Lebrazi H, Sabri M, Saile R. Usefulness of lipid ratios and atherogenic index of plasma in obese Moroccan women with or without metabolic syndrome. Ethn Dis. 2014;24(2):207-212. PubMed ID: 24804368

5. Tan MH, Johns D, Glazer NB. Pioglitazone reduces atherogenic index of plasma in patients with type 2 diabetes. Clin Chem. 2004;50(7):11841188. PubMed ID:15117857 doi:10.1373/clinchem.2004.031757
6. Kokkinos PF, Fernhall B. Physical activity and high density lipoprotein cholesterol levels: what is the relationship? Sports Med. 1999;28(5):307-314. PubMed ID: 10593643 doi:10.2165/ 00007256-199928050-00002

7. Skoumas J, Pitsavos C, Panagiotakos DB, et al. Physical activity, high density lipoprotein cholesterol and other lipids levels, in men and women from the ATTICA study. Lipids Health Dis. 2003;2:3. PubMed ID: 12852790 doi:10.1186/1476-511X-2-3

8. Mora S, Cook N, Buring JE, Ridker PM, Lee IM. Physical activity and reduced risk of cardiovascular events: potential mediating mechanisms. Circulation. 2007;116(19):2110-2118. PubMed ID: 17967770 doi:10.1161/CIRCULATIONAHA.107.729939

9. Warburton DE, Nicol CW, Bredin SS. Health benefits of physical activity: the evidence. CMAJ. 2006;174(6):801-809. PubMed ID: 16534088 doi:10.1503/cmaj.051351

10. Stranska Z, Matoulek M, Vilikus Z, Svacina S, Stransky P. Aerobic exercise has beneficial impact on atherogenic index of plasma in sedentary overweight and obese women. Neuro Endocrinol Lett. 2011;32(1):102-108. PubMed ID: 21407166

11. Ezeukwu AO, Agwubike EO, Uadia PO. Differential effects of continuous and interval exercise training on the atherogenic index of plasma in the non-obese young male. Zhonghua Minguo Xin Zang Xиe Hui Za Zhi. 2015;31(4):337-344.

12. Venojarvi M, Korkmaz A, Wasenius N, et al. 12 weeks' aerobic and resistance training without dietary intervention did not influence oxidative stress but aerobic training decreased atherogenic index in middle-aged men with impaired glucose regulation. Food Chem Toxicol. 2013; 61:127-135. PubMed ID: 23623841 doi:10.1016/j.fct.2013.04.015

13. Loprinzi PD. Dose-response association of moderate-to-vigorous physical activity with cardiovascular biomarkers and all-cause mortality: considerations by individual sports, exercise and recreational physical activities. Prev Med. 2015;81:73-77. PubMed ID: 26307435 doi:10.1016/j.ypmed.2015.08.014

14. Ainsworth BE, Haskell WL, Herrmann SD, et al. 2011 Compendium of physical activities: a second update of codes and MET values. Med Sci Sports Exercise. 2011;43(8):1575-1581. doi:10.1249/MSS. 0b013e31821ece12

15. Ainsworth BE, Haskell WL, Whitt MC, et al. Compendium of physical activities: an update of activity codes and MET intensities. Med Sci Sports Exercise. 2000;32(9 suppl):S498-S516. doi:10.1097/ 00005768-200009001-00009

16. Office of Disease Prevention and Health Promotion. Appendix 1. Translating Scientific Evidence about Total Amount and Intensity of Physical Activity into Guidelines. Available at: https://health.gov/ paguidelines/2008/appendix1.aspx

17. Earnest CP, Artero EG, Sui X, Lee DC, Church TS, Blair SN. Maximal estimated cardiorespiratory fitness, cardiometabolic risk factors, and metabolic syndrome in the aerobics center longitudinal study. Mayo Clin Proc. 2013;88(3):259-270. doi:10.1016/j.mayocp. 2012.11.006

18. Watt MJ, Heigenhauser GJ, Dyck DJ, Spriet LL. Intramuscular triacylglycerol, glycogen and acetyl group metabolism during $4 \mathrm{~h}$ of moderate exercise in man. $J$ Physiol. 2002;541(Pt 3):969-978. doi:10.1113/jphysiol.2002.018820

19. Dobiasova M. Atherogenic index of plasma $[\log ($ triglycerides/HDLcholesterol)]: theoretical and practical implications. Clin Chem. 2004;50(7):1113-1115. PubMed ID: 15229146 doi:10.1373/ clinchem.2004.033175

20. Dobiasova M, Frohlich J, Sedova M, Cheung MC, Brown BG. Cholesterol esterification and atherogenic index of plasma correlate with lipoprotein size and findings on coronary angiography. $J$ Lipid Res. 2011;52(3):566-571. PubMed ID: 21224290 doi:10.1194/jlr.P011668 